\title{
Electroless Pb Monolayer Deposition - Prelude for Further Advances in Catalyst Monolayer Synthesis via Surface Limited Redox Replacement Reaction
}

\author{
Kamyar Ahmadi ${ }^{2}$, Nikhil Dole ${ }^{1}$,Dongjun $\mathrm{Wu}^{1}$, Taha Salavati-Fard ${ }^{3,4}$, Lars. C. Grabow ${ }^{2,3,4}$, Francisco Carlos \\ Robles Hernandez ${ }^{5}$ and Stanko R. Brankovic ${ }^{1,2,3,{ }^{*}}$ \\ ${ }^{1}$ Electrical and Computer Engineering Department, University of Houston, Houston TX 77204 \\ ${ }^{2}$ Material Science and Engineering Program, University of Houston, Houston TX 77204 \\ ${ }^{3}$ William A. Brookshire Department of Chemical and Biomolecular Engineering, University of Houston, \\ Houston, TX 77204 \\ ${ }^{4}$ Texas Center for Superconductivity at the University of Houston (TcSUH), Houston, TX 77204 \\ ${ }^{5}$ Collge of Technology, University of Houston, Houston TX 77204
}

\begin{abstract}
*Corresponding author:
Stanko R. Brankovic

Department of Electrical and Computer Engineering, University of Houston, 77204

Email: SRBrankovic@uh.edu
\end{abstract}

\section{Supplemental Material}

\section{Experimental Details}

The starting $\mathrm{Au}(111), \mathrm{Pt}(111), \mathrm{Ru}(0001)$ were disk with diameter $10 \mathrm{~mm}$ and $3 \mathrm{~mm}$ thickness, (Monocrystals Company). They were prepared using mechanical polishing, electropolishing ( $\mathrm{Au}$ ) and hydrogen flame annealing ( $\mathrm{Au}$ ) or induction annealing ( $\mathrm{Pt}$ and $\mathrm{Ru}$ ) in inert atmosphere. This routinely yielded a highly reflective mirror-like surface with very reproducible $\mathrm{Pb}$ UPD voltammetry. The $\mathrm{Cu}<111>$ textured polycrystalline substrates were 100-200 nm thick films deposited on $\mathrm{SiO}_{2} / \mathrm{Si}$ wafer which were reduced in $\mathrm{H}_{2}+\mathrm{CO}$ gas mixture at $250{ }^{\circ} \mathrm{C}$ for one hour before the experiments. All solutions for $\mathrm{Pb}$ monolayer deposition and SLRR reaction were prepared with high purity grade chemicals such as $\mathrm{PbO}$, $\mathrm{VCl}_{2}, \mathrm{HClO}_{4}$ and $\mathrm{H}_{2} \mathrm{PdCl}_{4}, \mathrm{H}_{2} \mathrm{PtCl}_{4}$ (99.999\%, Alfa Aaeser, Merck) and $>18.2 \mathrm{M} \Omega$ ultra-pure water (Millipore Direct Q-UV with Barnstead A1007 pre-distillation unit). Before each experiment, solutions were deaerated for at least one hour with ultrapure nitrogen in order to minimize the concentration of dissolved oxygen from air. All experiments are performed using ultraclean glassware and oxygen-free environment. The volume of the electrochemical cell was $0.150 \mathrm{dm}^{-3}$ while the amount/volume of the reaction solution was standardized to $0.1 \mathrm{dm}^{-3}$ for each experiment. The volume of the solution during electrochemical quartz microbalance (ECQMB) studies and during insitu scanning tunneling microscopy (STM) studies was $0.001 \mathrm{dm}^{3}$. The quartz microbalance sample was a $50 \mathrm{~nm}$ thick gold film deposited on $2 \mathrm{~nm} \mathrm{Cr}$ seed on quartz disk with resonant frequency of $\approx 6 \mathrm{kHz}$. All potentials in the text are presented vs. siver-siver chloride reference electrode $(\mathrm{Ag} / \mathrm{AgCl} / 1.0 \mathrm{M} \mathrm{KCl} ; \mathrm{E}=0.235 \mathrm{~V}$ vs. SHE). The electrochemical studies were performed using BAS Epsilon system, while ECQMB experiments were performed using Metrohm Autolab PGSTAT 12 with integrated ECQMB module. The STM and AFM images were recorded using Nanoscope $V$ controller with multimode scanner unit (Veeco instruments). The solution for e-less $\mathrm{Pb} \mathrm{ML}$ deposition was prepared by mixing two volume part of the solution containing $\mathrm{Pb}^{2+}$ ions and one volume part of the solution containing $\mathrm{V}^{2+}$ ions. Both solutions contained $0.1 \mathrm{M} \mathrm{HClO}_{4}$ as a background electrolyte. 


\subsection{Experimental Routine}

All deposition experiment and the open circuit potential (OCP) measurements during the e-less $\mathrm{Pb} \mathrm{ML}$ deposition were performed in $\mathrm{N}_{2}$ filled glove box using either $\mathrm{Au}(111), \mathrm{Pt}(111), \mathrm{Ru}(0001)$ or $\mathrm{Cu}$ polycrystalline films as substrates. In the case of $\mathrm{Au}, \mathrm{Pt}$ and $\mathrm{Ru}$ single crystals, the hanging meniscus electrode configuration was used. In the case of $\mathrm{Cu}$ films, the samples were simply immersed into the $\mathrm{Pb}^{2+}$ containing solution. The entire experiment involved three steps. They are briefly explained below:

Step one: First, the cyclic voltammetry measurements were performed to verify the quality of the surface and characteristics of the Pb UPD process on a given surface ( $\mathrm{Au}$ or $\mathrm{Cu}$ ).

Step two: The potential is switched to the open circuit and OCP transients are recorded during the addition of $\mathrm{V}^{2+}$ containing aliquot. Typical length of the OCP measurements was between 50-100 seconds.

Step three: The sample with deposited e-less $\mathrm{Pb} \mathrm{ML}$ is transferred to a well de-aerated $0.1 \mathrm{M}$ $\mathrm{HClO}_{4}$ solution and the linear sweep is performed in anodic direction to strip the $\mathrm{Pb} \mathrm{ML}$ and record the stripping charge.

The same experimental routine is also performed during ECQMB measurements, except the third step was omitted.

\section{DFT Calculations}

All periodic density functional theory (DFT) calculations were carried out with the Vienna ab-initio simulation package (VASP) $)^{1-3}$ version 5.4.1 and the atomic simulation environment (ASE). ${ }^{4}$ Exchange and correlation effects were taken into account using the van der Waals corrected BEEF-vdW density functional which uses the Bayesian error estimation method to calculate energies. ${ }^{5}$ The wave function of the valance electrons was expanded into a plane wave basis set with an energy cutoff of $600 \mathrm{eV}$, while the core electrons were represented by the projector augmented wave function (PAW) method. ${ }^{6,7} \mathrm{~A}$ Gaussian-smearing scheme with $k_{B} T=0.1 \mathrm{eV}$ was used to treat discontinuities at the Fermi level, and the total energy was extrapolated to $k_{B} T=0.0 \mathrm{eV}$. Self-consistent field calculations were performed until an accuracy of $10^{-5} \mathrm{eV}$ in energy was achieved. Optimizations were performed with the conjugategradient algorithm, with a force threshold of $0.02 \mathrm{eV} / \AA ̊$. For $\mathrm{V}(\mathrm{OH})_{2}$ gas-phase calculations, narrower Gaussians with $k_{B} T=0.01 \mathrm{eV}$ and a tighter energy threshold of $10^{-6} \mathrm{eV}$ were used. In addition, A vacuum of $15 \AA$ and dipole corrections in all three dimensions were considered. For bulk systems, a $7 \times 7 \times 7$ k-points mesh was used to sample the reciprocal spaces. Slabs composed of five atomic layers were made using either $2 \times 2$ or $4 \times 4$ unit cells for which a $3 \times 3 \times 1 \mathrm{k}$-points mesh was found to be sufficient for sampling the reciprocal spaces. The two bottom atomic layers were kept frozen while the rest of the system was free to relax. A vacuum of $10 \AA$ was added to both top and bottom of the slab systems to avoid any interactions with periodically repeated images in the z-direction. Corrections to cancel out the long-range dipole-dipole interaction, in the z-direction, were also included.

(1) Kresse, G.; Hafner, J. Ab Initio Molecular-Dynamics Simulation of the Liquid-Metal-Amorphous- 
Semiconductor Transition in Germanium. Phys. Rev. B 1994, 49 (20), 14251.

(2) Kresse, G.; Hafner, J. Ab Initio Molecular Dynamics for Liquid Metals. Phys. Rev. B 1993, 47 (1), 558.

(3) Kresse, G.; Furthmüller, J. Efficiency of Ab-Initio Total Energy Calculations for Metals and Semiconductors Using a Plane-Wave Basis Set. Comput. Mater. Sci. 1996, 6 (1), 15-50.

(4) Larsen, A. H.; Mortensen, J. J.; Blomqvist, J.; Castelli, I. E.; Christensen, R.; Dułak, M.; Friis, J.; Groves, M. N.; Hammer, B.; Hargus, C. The Atomic Simulation Environment-a Python Library for Working with Atoms. J. Phys. Condens. Matter 2017, 29 (27), 273002.

(5) Wellendorff, J.; Lundgaard, K. T.; Møgelhøj, A.; Petzold, V.; Landis, D. D.; Nørskov, J. K.; Bligaard, T.; Jacobsen, K. W. Density Functionals for Surface Science: Exchange-Correlation Model Development with Bayesian Error Estimation. Phys. Rev. B 2012, 85 (23), 235149.

(6) Blöchl, P. E. Projector Augmented-Wave Method. Phys. Rev. B 1994, 50 (24), 17953.

(7) Kresse, G.; Joubert, D. From Ultrasoft Pseudopotentials to the Projector Augmented-Wave Method. Phys. Rev. B 1999, 59 (3), 1758.

\section{HRTEM}

The TEM images were collected on a JEOL 2100F TEM operated at $200 \mathrm{kV}$, under the bright field conditions. The samples were prepared on a holey carbon Cu grid of 300 mesh. 